\title{
THREE SIMPLE AIDS IN REFRACTION WORK
}

BY

F. W. G. Smith, M.A., M.D., M.CH.

LONDON

THESE instruments have no scientific merit but my excuse for describing them is that I have found them useful in the routine examination of patients.

\section{Finger Clip Lenses}

The principle of this method is to carry on two fingers the four lenses most used in refraction cases, while being of such a size that they will not interfere with all ordinary movements of the hands.

Two spherical lenses of $2 \mathrm{cms}$. diameter and of opposite power are carried on an arm projecting from a split sleeve which is made of blackened soft metal in order that the instrument can be bent to suit the individual user. I have found that plus and minus $0 \cdot 25 \mathrm{D}$. and $0 \cdot 50 \mathrm{D}$. spheres are the most useful lenses but other powers could be obtained. The strength of the pair of lenses is

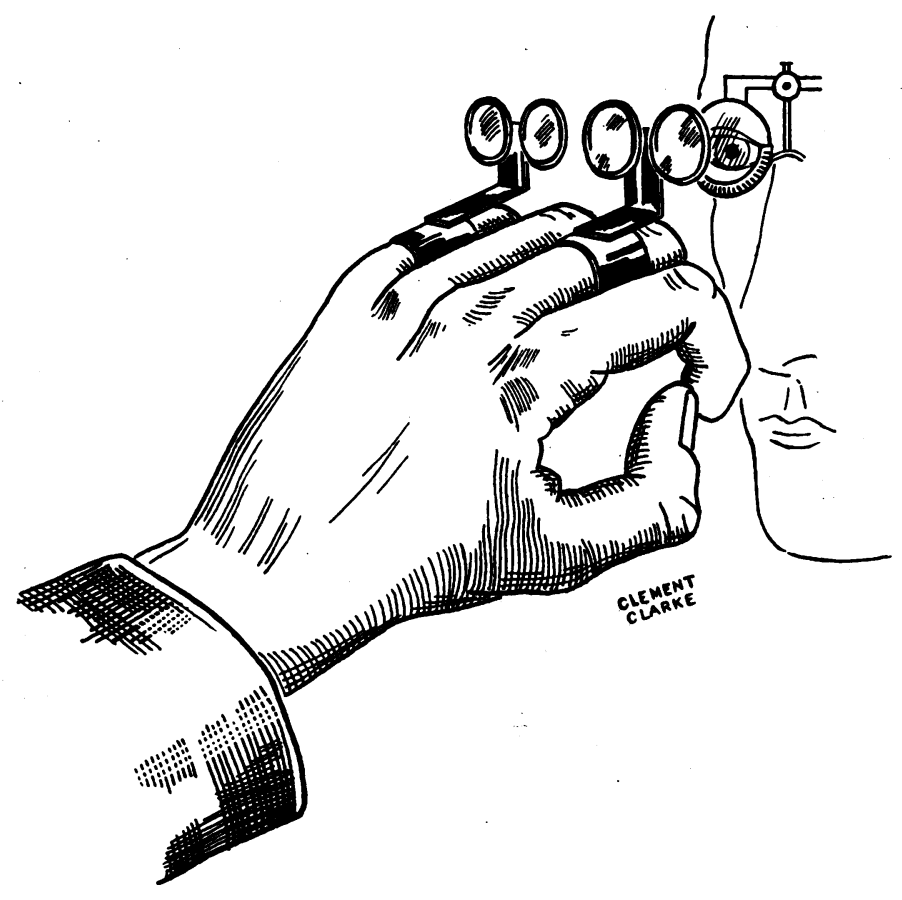


stamped on the holder and the rim of the minus lens is painted white.

The clips are slipped on to the proximal phalanges of the middle and little fingers of the left hand, or whichever the user finds more convenient, and retained on the hand until the series of patients is finished. The hands can be washed with the lenses on the fingers.

When in use the lenses are brought successively against the glass in the trial frame but the hand is usually held more obliquely and the wrist more curved than is shown in the illustration.

I have found, in cylinder retinoscopy, that the strength of the sphere and cylinder can be gauged rapidly with few changes of lenses in the trial frame and that subjectively the effect of a quick contrast makes the choice of the correct lens easier for the patient.

The labour and time expended on each refraction case is appreciably reduced, in my experience, when these lenses are employed.

\section{Children's Distance Test}

The type is made of two pieces of strong cardboard folding into 30 by 24 inches on which are six charts, three of which are visible at one time. The left chart has a Maddox single row of letters, the middle one is of simple coloured objects subtending about $6 / 60$ to $6 / 9$ and the right hand one has the full rows of letters. Red and green spots one inch in diameter are placed at each end of the $6 / 9$ lines in all charts. The other three charts are similar but have different lettering. The folding side-pieces add to the

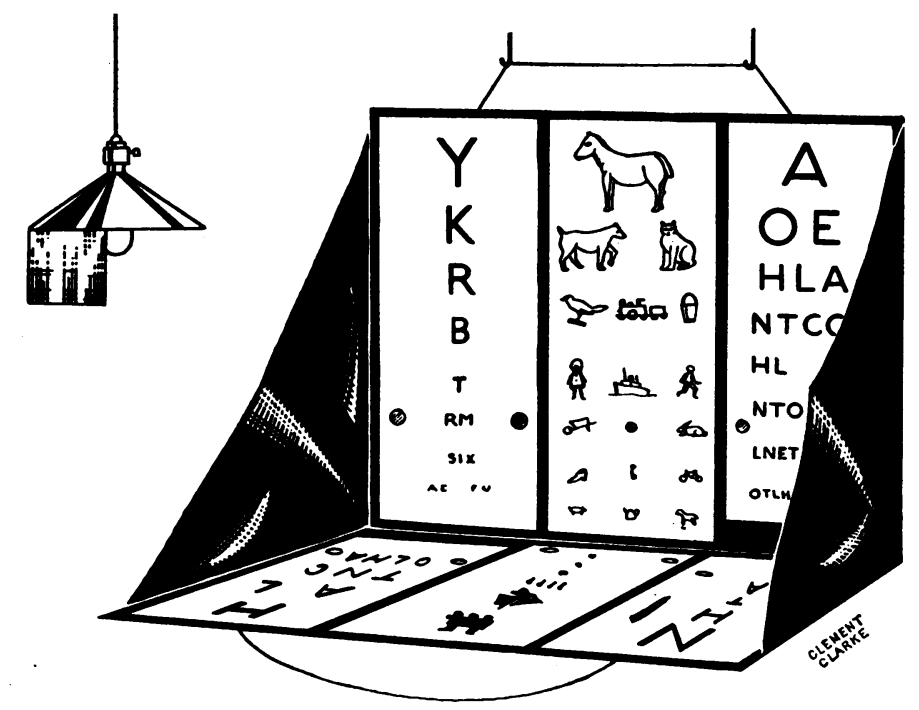


strength and aid in obscuring the lower letters from children awaiting examination. It is only necessary to invert the type to expose the other set of charts. A lamp hanging from the ceiling above the types, with the direct light shaded off the patient, gives satisfactory illumination.

After the refraction has been done the child is asked to read the right or left chart and at the same time is tapped on the corresponding cheek. If the child appears intelligent he is asked at once to read the letters between the coloured spots. A child who is suspected of knowing the letters can be confused by switching him from one chart to the other. The coloured pictures are pointed out with a stick for those children who cannot read their letters.

I have found these types especially convenient where clinics are held in rooms which are not fitted for eye work.

\section{The Examination of Patients confined to bed}

I was led to evolve this method as I found that I could not obtain really satisfactory results by other means. Some of the apparatus is home-made and possibly unduly clumsy but all of it, with a set of trial lenses, can be carried in a suit case.

The essentials are a mirror, a folding metal music stand and a test type box somewhat similar to the Drummond Currie test case but having a Maddox spot light.
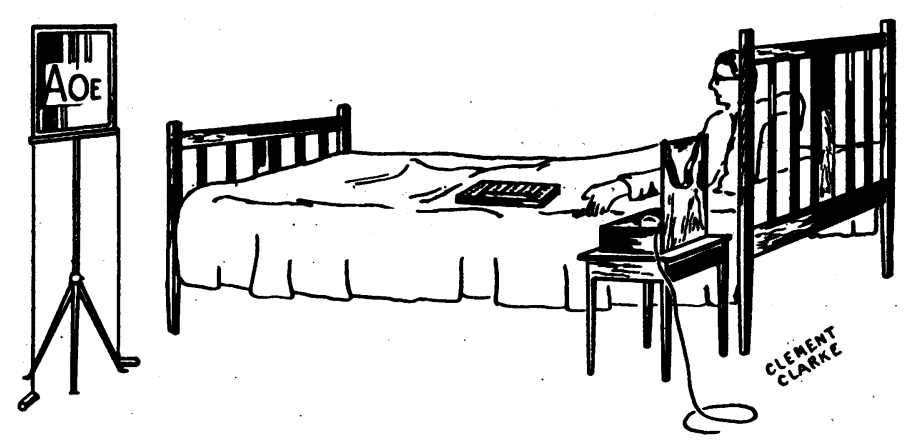

The mirror is hung on the music stand which is readily adjustable as regards height and inclination. A pair of weighted strings anchor the mirror when it is set and prevent fine oscillations.

The apparatus can be put up in five minutes. I have found that the reversed method is necessary as it is rarely possible to get the full direct distance outside a hospital ward.

I am indebted to Messrs. Clement Clarke for the sketches and for carrying out my designs as regards the finger clip lenses and the children's test-type. 